12

\title{
Прецизионные методы измерения коэффициента отражения зеркальных покрытий
}

\author{
(ㄱ С.А. Бельков, В.Н. Деркач, С.Ю. Головкин, А.С. Губкин , В.Ю. Добикова, В.О. Лащук, \\ Д.В. Сизмин, В.А. Щеников
}

Российский Федеральный Ядерный Центр - ВНИИЭФ, 607188 Саров, Нижегородская обл., Россия

ฯ e-mail: oefimova@otd13.vniief.ru

Поступила в редакцию 29.09.2020 г.

В окончательной редакции 28.12.2020 г.

Принята к публикации 10.05.2021 г.

\begin{abstract}
Рассмотрены методы определения коэффициентов отражения зеркальных покрытий оптических деталей, основанные на многократном отражении излучения от измеряемой поверхности и контроле величин регистрируемых сигналов, обеспечивающие абсолютную погрешность измерения $<0.1 \%$. Проведена их экспериментальная аттестация, подтвердившая высокую точность таких измерений.
\end{abstract}

Ключевые слова: коэффициент отражения, мощные лазерные установки, погрешность измерения.

DOI: $10.21883 /$ OS.2021.09.51349.246-21

\section{Введение}

Одним из важных требований к конструктивным элементам современных лазерных установок является высокая однородность покрытий оптических элементов и соответствие их коэффициента отражения или пропускания заданным параметрам. В ряде случаев, когда количество отражающих и просветляющих покрытий является большим, невысокое качество изготовления покрытий может приводить к существенным и некомпенсируемым потерям в энергии и мощности излучения, появлению амплитудной и фазовой модуляции пучка. Для установок с большим количеством каналов [1-3] этот фактор может также вызывать дисбаланс энергетических параметров, для устранения которого потребуется создание сложного адаптивного управления.

В этой связи разработка „быстрых“ и высокоточных инструментов аттестации покрытий по коэффициенту отражения является актуальной. В данной работе рассматриваются только методы измерения коэффициента отражения, однако эти же методы могут быть адаптированы и для измерения пропускания.

Обычно для повышения точности измерения коэффициентов отражения используют многократные отражения от образца $[4,5]$, чаще всего используется схема с двукратным отражением как наиболее простая. Исторически первым вариантом схемы с двукратным отражением были так называемая VW-схема [6,7] и ее аналоги [8]. Общим недостатком таких схем является то, что 2 отражения, используемые для измерения коэффициента отражения не равнозначны: в случае VWсхемы пучок отражается от разных точек образца, а в другом варианте два пучка приходят в одну точку, но под разными углами. Впоследствии вместо VW-схемы была предложена IV-схема, которая лишена указанных недостатков [9].
Запатентованные авторами работы методы [10,11] являются дальнейшим развитием IV схемы и схемы с многократным (более 2 раз) количеством отражений. Отличие от ранее используемых методов заключается в том, что для повышения точности измерений предлагается выравнивать величину сигналов на разных этапах измерения, тем самым избавляясь от систематической ошибки, связанной с нелинейностью передаточных характеристик измерительных приборов.

\section{Измерение коэффициента отражения с контролем величин измеряемых сигналов}

Поскольку коэффициент отражения диэлектрических покрытий зависит от угла падения излучения, а также от того, как излучение поляризовано, в контексте измерений имеет смысл говорить лишь о коэффициенте отражения для конкретного угла и для одного из двух направлений поляризации. Поэтому здесь и далее под абсолютным значением коэффициента отражения понимается именно коэффициент отражения, характеризующий деталь при заданном угле падения и направлении поляризации, выраженный в абсолютных единицах.

Принципиальная схема измерения коэффициентов отражения, используемая авторами работы [10], представлена на рис. 1. Схема включает в себя измеряемое и вспомогательное зеркала, а процедура измерения состоит из двух этапов. На первом (рис. 1, $a$ ) измерение осуществляют без измеряемой детали. На втором, в соответствии с рис. $1, b$, в тракт помещается исследуемая деталь, а вспомогательное зеркало перемещается в новое положение, но при этом размещается таким образом, чтобы длина оптического пути от первого клина до измерителя $D_{2}$ оставалась неизменной. В обоих 

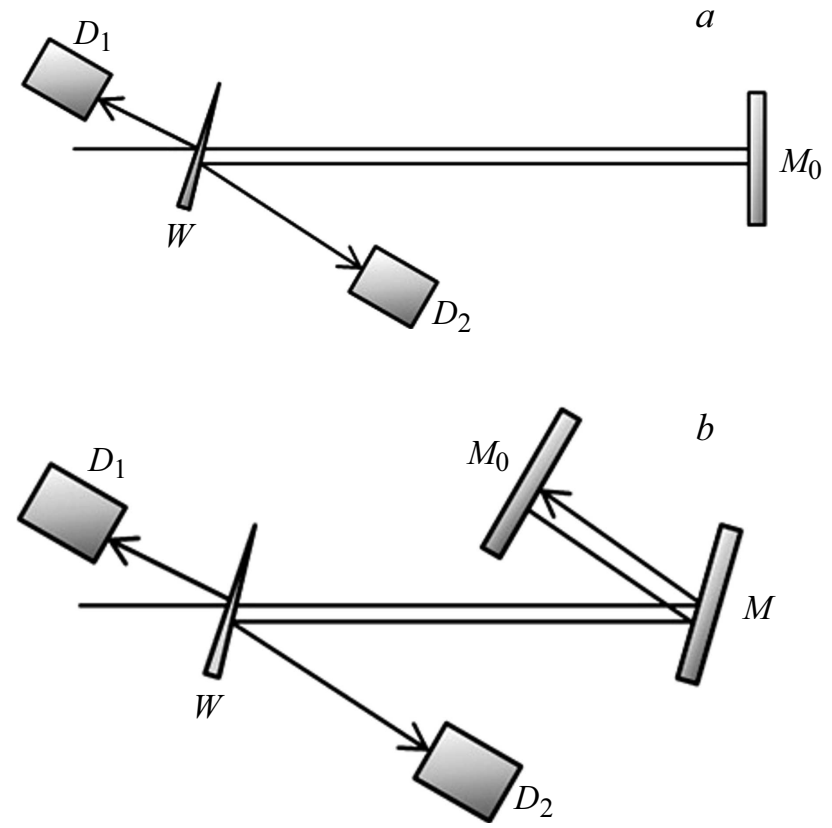

Рис. 1. Схема измерений с двукратным отражением излучения без образца $(a)$ и с образцом $(b): M_{0}$ - вспомогательное зеркало, $W-$ светоделительный клин, $M-$ измеряемое зеркало, $D_{1}$ - измерительный канал для регистрации излучения, падающего на измеряемое зеркало, $D_{2}-$ измерительный канал для регистрации излучения, отраженного от измеряемого зеркала.

случаях вспомогательное зеркало располагается нормально по отношению к падающему излучению, за счет этого коэффициент отражения данного зеркала не изменяется при переходе от первого этапа измерения к второму. В результате точность измерений не зависит от характеристик вспомогательного зеркала. Измерения, выполняемые на первом и на втором этапах, отличаются тем, что во втором случае излучение дважды отражается от измеряемой детали, коэффициент отражения детали составляет:

$$
R=\sqrt{\frac{P_{1}}{P_{2}} \frac{P_{2}^{\prime}}{P_{1}^{\prime}}}
$$

где $P_{1}$ и $P_{2}-$ величины сигналов в первом и втором каналах регистрации на первом, $P_{1}^{\prime}$ и $P_{2}^{\prime}$ - втором этапе измерений.

Представим выражение для погрешности измерений в виде формулы с отдельным вкладом систематической и случайной составляющей:

$$
\begin{aligned}
\delta R= & \delta R_{r}+\delta R_{s}=\frac{1}{2}\left(\frac{\Delta P_{1 s}+\Delta P_{1 r}}{P_{1}}-\frac{\Delta P_{1 s}^{\prime}+\Delta P_{1 r}^{\prime}}{P_{1}^{\prime}}\right. \\
& \left.+\frac{\Delta P_{2 s}^{\prime}+\Delta P_{2 r}^{\prime}}{P_{2}^{\prime}}-\frac{\Delta P_{2 s}+\Delta P_{2 r}}{P_{2}}\right),
\end{aligned}
$$

где символы $s$ и $r$ в индексах относятся к слагаемым погрешности систематического и случайного типа.

К систематической следует отнести погрешность, связанную с изменением передаточной функции измери- тельного прибора при изменении величины регистрируемого сигнала. Выделим несколько независимых источников случайной погрешности и перечислим здесь основные. Это ограниченный набор статистики измерений в условиях переменной мощности цифруемого сигнала и неточность юстировки схемы регистрации второго из каналов на разных этапах измерений, выражающаяся в изменении размера пучка и области попадания на приемную площадку прибора, а также изменении коэффициента отражения вспомогательного зеркала. Способы уменьшения погрешностей разного типа требуют проведения различных мероприятий и будут далее рассматриваться нами в отдельности.

Измерения в двух измерительных каналах проводились с частотой около $10 \mathrm{~Hz}$ поочередно, т. е. второй датчик запускается через $0.1 \mathrm{~s}$ после первого, еще через $0.1 \mathrm{~s}$ снова запускается первый и т.д. Характерная мощность лазера, используемого для проведения измерений, устанавливалась в диапазоне от 200 до $300 \mathrm{~mW}$.

Поскольку мощность лазера может меняться во время измерения, описанный выше алгоритм работы датчиков приводит к тому, что величина $P_{2} / P_{1}$ определяется с некоторой погрешностью $\varepsilon_{1}$. Для уменьшения данной погрешности использовались усредненные на большом временном интервале значения сигналов $\left\langle P_{1}\right\rangle$ и $\left\langle P_{2}\right\rangle$. Считалось, что сигнал, усредненный по интервалу измерений в $N_{0}=2 \cdot 10^{4}$ отсчетов, является точным. Количество отсчетов $N$, при котором выполняется условие:

$$
\left|\left\langle\frac{P_{2}}{P_{1}}\right\rangle_{N}-\left\langle\frac{P_{2}}{P_{1}}\right\rangle_{N_{0}}\right| \leq \varepsilon_{1},
$$

было определено экспериментально. Измерения проводились по схеме, представленной на рис. $1, a$, в результате было получено, что требование $\varepsilon_{1} \leq 2 \cdot 10^{-4}$ выполняется для $N=100$ при условии стабилизации сигнала источника излучения на уровне СКО $\left(P_{1}\right)<10^{-5} \mathrm{~W}$. В последующем изложении всегда будем понимать, что определенные выше требования к проведению измерений выполнялись.

Другим источником случайной ошибки является переюстировка схемы при переходе с этапа на этап. Для однозначности юстировки измерительных схем в них внесены светоделительные элементы, отводящие часть излучения в каналах регистрации на камеры, фиксирующие стабильность трассировки излучения. Была проведена серия из $M=10$ независимых измерений величины $\left\langle\frac{P_{2}}{P_{1}}\right\rangle_{N}$ по схеме, представленной на рис. $1, b$, перед каждым измерением проводилась процедура повторной юстировки вспомогательного зеркала. Получено, что максимальное отклонение от среднего по серии измерений составляет $\approx 0.05 \%$. Полученный результат включает в себя оба источника случайной ошибки и может служить оценкой ее полной величины. Оценим далее полную величину погрешности измерения коэффициента отражения $\delta R_{r}$, обусловленную случайной ошибкой измерения $\left\langle\frac{P_{2}}{P_{1}}\right\rangle$. Из (2) следует

$$
\delta R_{r}=\frac{1}{2}\left(\left|\delta\left(\frac{P_{1}}{P_{2}}\right)_{r}\right|+\left|\delta\left(\frac{P_{2}^{\prime}}{P_{1}^{\prime}}\right)_{r}\right|\right) \leq 5 \cdot 10^{-4} .
$$


Рассмотрим далее прием, примененный нами для ограничения систематической составляющей погрешности измерения и базирующийся на выбранной 2-этапной процедуре измерения.

Предположим, что изменение сигнала между этапами измерения является малой величиной в обоих измерительных каналах: $P_{1}^{\prime}=P_{1}+\varphi_{1}$, где $\left|\frac{\varphi_{1}}{P_{1}}\right| \ll 1$ и $P_{2}^{\prime}=P_{2}+\varphi_{2}$, где $\left|\frac{\varphi_{2}}{P_{2}}\right| \ll 1$. На практике это означает, что источник излучения работает стабильно, а изменения в пропускании схемы второго измерительного канала не существенны, т. е. измеряемое зеркало имеет коэффициент отражения близкий к ста процентам. Тогда, можно ожидать, что систематические погрешности измерений „примерно равных“ сигналов в каждом из каналов на разных этапах отличаются незначительно, т. е. $\Delta P_{1 s} \cong \Delta P_{1 s}^{\prime}$ и $\Delta P_{2 s} \cong \Delta P_{2 s}^{\prime}$. Это позволяет учесть знак погрешности в выражении (2) и свести систематическую составляющую погрешности коэффициента отражения к виду

$$
\begin{aligned}
& \delta R_{s}=\frac{1}{2}\left(\frac{\Delta P_{1 s}}{P_{1}}-\frac{\Delta P_{1 s}^{\prime}}{P_{1}^{\prime}}+\frac{\Delta P_{2 s}^{\prime}}{P_{2}^{\prime}}-\frac{\Delta P_{2 s}}{P_{2}}\right) \\
& \cong \frac{1}{2}\left(\frac{\Delta P_{1 s}}{P_{1}}-\frac{\Delta P_{1 s}\left(P_{1}-\varphi_{1}\right)}{P_{1}^{2}}+\frac{\Delta P_{2 s}\left(P_{2}-\varphi_{2}\right)}{P_{2}^{2}}-\frac{\Delta P_{2 s}}{P_{2}}\right) \\
& \cong \frac{1}{2}\left\{\left|\frac{\Delta P_{1 s}}{P_{1}}\right|\left|\frac{\varphi_{1}}{P_{1}}\right|+\left|\frac{\Delta P_{2 s}}{P_{2}}\right|\left|\frac{\varphi_{2}}{P_{2}}\right|\right\}
\end{aligned}
$$

где $\frac{\Delta P_{1 s}}{P_{1}}$ и $\frac{\Delta P_{1 s}}{P_{1}}-$ погрешности систематических измерений в обоих каналах.

Из выражения (5) получается, что при

$$
\frac{\Delta P_{1 s}}{P_{1}} \cong \frac{\Delta P_{1 s}}{P_{1}} \leq 3 \cdot 10^{-2}
$$

и

$$
\left|\frac{\varphi_{1}}{P_{1}}\right| \cong\left|\frac{\varphi_{2}}{P_{2}}\right|<10^{-2}
$$

систематическая ошибка измерения коэффициента отражения $\delta R_{s}<3 \cdot 10^{-4}$.

Таким образом, с учетом определенного выше регламента и комплекса мер, направленных на увеличение точности измерений, показано, что суммарная погрешность определения коэффициента отражения составляет величину, меньшую $10^{-3}$.

При выводе (5) использовалось приближение, ограничивающее область применения методики измерения случаем аттестации зеркал с коэффициентом отражения, близким к ста процентам. Для расширения возможностей применяемого метода предложено вместо схемы на рис. 1, $a$ использовать схему, представленную на рис. 2. Стабильность сигнала в канале $D_{2}$ в случае испытания зеркал с меньшим коэффициентом отражения может быть достигнута за счет применения дополнительного элемента $E$, вводимого в схему на первом этапе и обеспечивающего равенство потерь на двух этапах измерения.

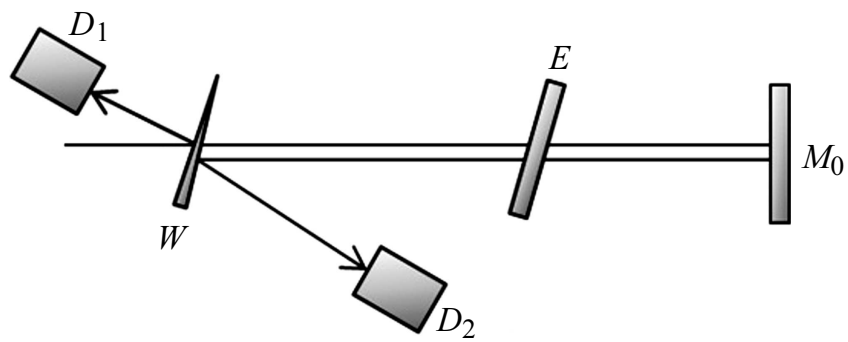

Рис. 2. Схема измерения с контролем величин регистрируемых сигналов. $E-$ эталонный элемент, остальные обозначения те же, что и на рис. 1.

В качестве элемента $E$ может быть использован эталон с хорошо известным показателем преломления и малыми коэффициентами поглощения и деполяризации, например, синтетический кварц с высоким показателем однородности материала. При таком выборе потери в мощности излучения определяются преимущественно френелевским отражением и регулируются за счет изменения угловой ориентации элемента, определяемой с высокой точностью. Двойное прохождение пучка через эталон обусловлено стремлением компенсировать поперечное смещение пучка при прохождении через элемент $E$ и обеспечить сохранение положения пучка на приемной площадке прибора в измерительном канале $D_{2}$ на разных этапах аттестации.

Коэффициент отражения определяется вместо (1) по формуле

$$
R=T \sqrt{\frac{P_{2}^{\prime}}{P_{2}} \frac{P_{1}}{P_{1}^{\prime}}},
$$

где $T$ - коэффициент пропускания элемента $E$ за один проход излучения. В этом случае относительная погрешность измерения коэффициента отражения примет вид

$$
\delta R \leq \frac{1}{2}|\delta P|\left\{\left|\frac{\varphi_{1}}{P_{1}}\right|+\left|\frac{\varphi_{2}}{P_{2}}\right|\right\}+\delta T .
$$

Полученное выражение отличается от (2) слагаемым $\delta T$. Проведем здесь оценку погрешности, вызванной этой добавкой. Пусть коэффициент отражения исследуемой детали $R=95 \%$. Для обеспечения равенства потерь на этапах измерений эталон должен быть расположен под углом $\varphi \approx 25.7^{\circ}$ к $p$-поляризованному излучению. Так как зависимость коэффициента отражения эталона от угла $R_{E}(\varphi)$ известна, можно оценить величину погрешности, связанную с погрешностью ориентации для заданного угла. Угол падения на эталон определяется по теореме косинусов через величину базовых расстояний $L_{i}$, формирующих треугольник с вершиной на эталонной детали. Для наших экспериментов характерна погрешность измерений $\delta L_{i}<0.05 \%$, что, в свою очередь, определяет погрешности измерения угла и пропускания эталона как $\Delta \varphi \approx 5 \cdot 10^{4} \mathrm{rad}$ и $\delta T \approx 0.004 \%$ соответственно. Полученное в результате оценки значение $\delta T$ на порядок меньше, чем погрешность в схеме 


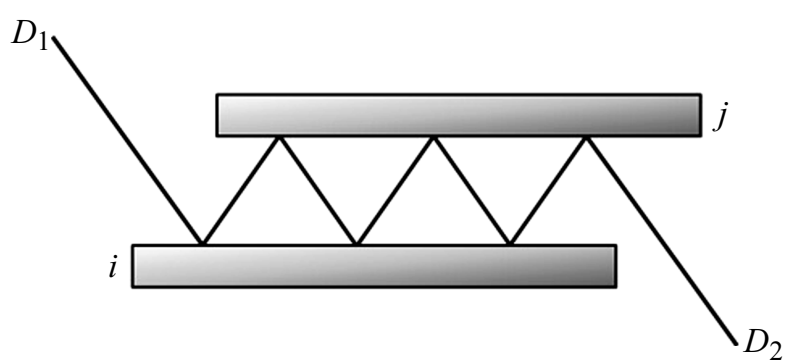

Рис. 3. Многократное отражение при прохождении зазора из двух деталей.

измерений без элемента $E$, и вносимая добавка не приводит к существенному увеличению погрешности $\delta R$. Следовательно, можно утверждать, что использование эталонного элемента позволяет расширить диапазон измеряемых значений коэффициента отражения, сохранив при этом высокую точность.

\section{Измерение коэффициента отражения с многократным отражением излучения}

Рассмотрим далее другой вариант определения коэффициентов отражения с повышенной (относительно стандартных методов) точностью измерений - это способ, при котором лазерное излучение отражается от исследуемой детали многократно. Такой способ уменьшает погрешность измерения, в то же время усредняя измеряемую величину по апертуре исследуемой детали. Ранее авторами работы был предложен новый способ [11] на основе многократного отражения излучения от тестируемой детали, обеспечивающий высокую точность измерений. Принципиальная схема таких измерений представлена на рис. 3 .

Измерения проводятся по одинаковой оптической схеме с последовательной заменой зеркал:

- этап 1: образцы № 1 и № 2;

- этап 2: образцы № 3 и № 2;

- этап 3: образцы № 3 и № 1.

На каждом этапе излучение отражается от каждого из покрытий n раз, при этом осуществляется регистрация падения величины сигнала в результате многократного отражения.

Коэффициент отражения образца $z$ выражается следующим образом:

$$
R_{z}=\left(\frac{P_{2}}{P_{1}}\right)^{i / 2 n}\left(\frac{P_{1}^{\prime}}{P_{2}^{\prime}}\right)^{j / 2 n}\left(\frac{P_{2}^{\prime \prime}}{P_{1}^{\prime \prime}}\right)^{k / 2 n}
$$

где $P_{1}$ и $P_{2}-$ мощности измеряемых сигналов в первом и втором каналах регистрации, количество штрихов соответствует этапу измерений, а $i, j, k-$ параметры, определяющие вид выражения для каждого образца. Для первого образца $\{i, j, k\}=(1,1,1)$, для второго $(1,-1,-1)$ и для третьего - $-1,-1,1)$.
Выражение для относительной погрешности измерения коэффициента отражения первого зеркала можно представить в виде

$$
\delta R_{1} \cong \frac{1}{2 n}\left(-\frac{\Delta P_{1}}{P_{1}}+\frac{\Delta P_{1}^{\prime}}{P_{1}^{\prime}}-\frac{\Delta P_{1}^{\prime \prime}}{P_{1}^{\prime \prime}}+\frac{\Delta P_{2}}{P_{2}}-\frac{\Delta P_{2}^{\prime}}{P_{2}^{\prime}}+\frac{\Delta P_{2}^{\prime \prime}}{P_{2}^{\prime \prime}}\right)
$$

Для случая $\left|\delta P_{1}\right| \cong\left|\delta P_{1}^{\prime}\right| \cong\left|\delta P_{1}^{\prime \prime}\right|$ и $\left|\delta P_{2}\right| \cong\left|\delta P_{2}^{\prime}\right| \cong\left|\delta P_{2}^{\prime \prime}\right|$ для любого из трех зеркал выражение (9) можно представить в виде

$$
\delta R_{i} \cong \frac{3}{2 n}\left(\left|\delta P_{1}\right|+\left|\delta P_{2}\right|\right) .
$$

При погрешности измерения сигналов $3 \%$ и количестве отражений $\mathrm{n}=100$ погрешность измерения составит $\delta R_{i} \cong 0.1 \%$. Отличительной чертой данного метода (относительно основанного на контроле величин измеряемых сигналов) является интегральный характер измерений.

\section{Экспериментальные данные}

С целью экспериментальной проверки предлагаемых методик были проведены 3 серии из 5 независимых измерений по двум предложенным схемам (рис. 2,3). В качестве исследуемой была выбрана плоская оптическая деталь с зеркальным покрытием. Геометрические параметры детали: диаметр $100 \mathrm{~mm}$, толщина $10 \mathrm{~mm}$. Угол падения излучения, для которого нанесено покрытие, составлял $45^{\circ}$. Длина волны лазерного излучения - $527 \mathrm{~nm}$, тип поляризации - $p$. Измерения проводились именно в тех условиях, на которые рассчитано диэлектрическое покрытие образца. Однако схема экспериментального стенда позволяет установить произвольный угол падения излучения и выбрать любое направление поляризации, так как деталь располагается на поворотном столике, а перед измерительной схемой установлена призма-поляризатор. При проведении измерений погрешность установки угла измеряемой детали не превышала 5', а изменение коэффициента отражения, вызванное неточностью установки детали, считалось пренебрежимо малым.

В качестве эталонного элемента в схеме с контролем величин регистрируемых сигналов использовался образец синтетического кварца, изготовленный из материала Suprasil 1, диаметром $75 \mathrm{~mm}$, толщиной $12.9 \mathrm{~mm}$. Результаты многократных измерений коэффициента отражения с использованием эталонного элемента представлены на рис. 4, а. Штриховая линия - усредненное по серии значение, вертикальные - погрешности измерений. В измерении обеспечена погрешность $<0.1 \%$, максимальное отклонение измеренной величины от среднего значения для данной серии составляет менее $0.05 \%$.

Следующая серия измерений проводилась по схеме с многократным отражением, дополнительно использовались еще 2 зеркала, количество отражений от деталей составляло 30. Для такого количества отражений 

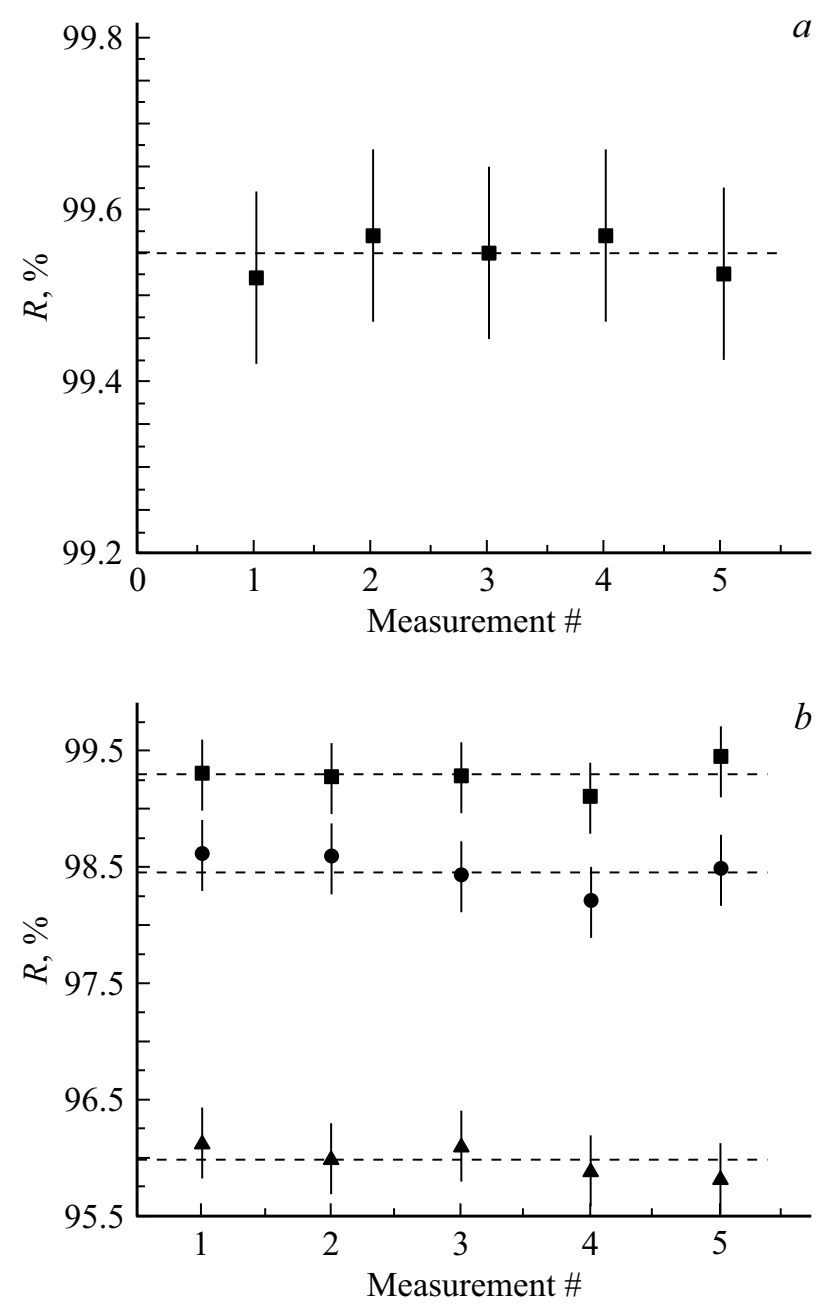

Рис. 4. Результаты измерений по схемам с контролем величин регистрируемых сигналов $(a)$ и с многократным отражением излучения $(b)$. Квадратные маркеры - результаты измерений детали № 1, круглые - детали № 2, треугольные - детали № 3.

относительная погрешность измерения коэффициента отражения, рассчитанная по формуле (10), составляет $\approx 0.3 \%$. Результаты измерений коэффициента отражения всех трех оптических деталей по схеме с многократным отражением представлены на рис. 4, $b$. Максимальное отклонение результатов измерения от соответствующего среднего значения для данной серии составляет около $0.2 \%$.

Отметим также, что разница в установленной величине коэффициента отражения, определенной двумя представленными способами, составляет $\approx 0.2 \%$. Полученная разница объясняется тем, что способ, использующий многократные отражения, измеряет среднее значение коэффициента отражения по апертуре детали, а также в целом более чувствителен к точности юстировки.

Дополнительно измерения были продублированы на спектрофотометре PhotonRT с паспортной относительной погрешностью $0.5 \%$. Значения максимального откло- нения, полученные разными методами, не превышают тех величин погрешности, которые были получены ранее в результате оценки.

\section{Заключение}

Рассмотрены различные способы измерения коэффициентов отражения зеркал с погрешностью $\leq 0.1 \%$, основанные на принципах:

- контроля величин регистрируемых сигналов;

- многократного отражения излучения от исследуемой поверхности.

Проведены испытания данных способов измерений на оптических деталях с малой световой апертурой. Получена погрешность измерений коэффициентов отражения $<0.1 \%$ по схеме с контролем величин сигналов и $<0.3 \%$ по схеме с многократным отражением излучения. Повторяемость результатов измерений составила соответственно $\leq 0.05 \%$ и $\leq 0.2 \%$.

Дальнейшее снижение погрешности измерений возможно в случае объединения предложенных способов.

\section{Конфликт интересов}

Авторы заявляют, что у них нет конфликта интересов.

\section{Список литературы}

[1] Гаранин С.Г., Зарещкий А.И., Илькаев Р.И. и др. // Квант. электрон. 2005. Т. 35. С. 299.

[2] Haynam C.A., Wegner P.J. et al. // Appl. Opt. 2007. V. 46. P. 3276.

[3] Nicolas O., Le Garrec B.J. // J. Phys.: Conf. Ser. 2008. V. 112. 032019.

[4] Павлюков А.К., Курт В.И. Патент РФ № 2467309, 2012.

[5] Левин Б.М. Авт. свид. № 151063, 1962.

[6] Bennet H.E., Koehler W.F. // J. Opt. Soc. Am. 1960. V. 50. P. 1.

[7] Archard M.A. // Workshop on Optical Property Measurement Techniques, Ispra, Italy 27-29 October 1987. Commission of the European Communities, May. 1988. 73.

[8] Волков В.Н., Порозов В.А. Авт. свид. № 411356, 1974.

[9] van Nijnatten P.A., de Wolf J.M.C., Schoofs I.J.E. // Directional reflection measurements on highly reflecting coatings, 7th International Conference on Coatings on Glass, Eindhoven, 2008.

[10] Головкин С.Ю. и др. Патент РФ № 2660398, 2018.

[11] Головкин С.Ю. и др. Патент РФ № 2643216, 2018. 